\title{
Cinematographic adaptation as support in FFL class: the case of Madame Bovary
}

\section{[Adaptation cinématographique comme support en classe de FLE : le cas de Madame Bovary]}

\author{
Valbona Gashi-Berisha - Lendita Gjikolli
}

DOI: 10.18355/XL.2019.12.03.10

\begin{abstract}
In the era of audio-visual, the literary text is difficult to understand, especially, the fact to be relevant to our students learning French as a second language, the understanding, however, continues to pose obstacles to the learning access of language and didactic knowledge.

Thanks to the Literature and Cinematography module, we could ascertain the importance of image education, both pedagogically and didactically. The visual and sound language transmitted by the cinema allows students to easily understand the target language. The cinema is an artistic and cultural medium capable of facilitating literary education in the XXI century.

The most complex and intriguing female character in French literature, Emma Bovary of Flaubert is the heroine who always returns to the honor of cinematography. Although Madame Bovary's adaptations tell the story of Emma's life, yet each director gives the character his vision, his interpretation.

This study will examine the power of film adaptations of Madame Bovary: Jean Renoir (1934), Vincente Minnelli (1949), Claude Chabrol (1990) and Sophie Barthes (2014).

Our paper consists of showing how to make a clear literary comparison about Madame Bovary's cinematographic adaptations as well as about the work and its reception.
\end{abstract}

Key words: adaptation, literature, cinema, support, teaching

\section{Résumé}

Á l'ère de l'audio-visuel, le texte littéraire est difficile à comprendre, en particulier, le fait qui doit être pertinent à nos étudiants apprenant le français comme langue seconde dont la compréhension continue toutefois de poser déjà des obstacles à l'accès aux connaissances langagières et didactiques.

Grâce au module Littérature et cinématographie, nous avons pu constater l'importance d'une éducation à l'image autant sur le plan pédagogique que sur le plan didactique. Le langage visuel et sonore que transmet le cinéma permet à l'étudiant de comprendre plus facilement la langue cible. Le cinéma représente un support artistique et culturel capable de faciliter l'enseignement littéraire au XXIème siècle.

Le personnage féminin le plus complexe et le plus intriguant de la littérature française, Emma Bovary de Flaubert est l'héroïne qui revient toujours à l'honneur de la cinématographie. Bien que les adaptations de Madame Bovary racontent effectivement la vie d'Emma, chaque réalisateur donne pourtant au personnage sa propre vision, son interprétation.

Cette étude se penchera sur la puissance des adaptations cinématographiques de Madame Bovary: Jean Renoir (1934), Vincente Minnelli (1949), Claude Chabrol (1990) et Sophie Barthes (2014). 
Notre travail consiste à montrer comment effectuer une comparaison littéraire, et à faire comprendre ce que les adaptations cinématographiques de Madame Bovary nous disent de l'œuvre et de sa réception.

Mots clés : adaptation, littérature, cinéma, support, enseignement

\section{Introduction : de la plume à la caméra}

Le cinéma, pareil tout comme le texte, fait partie des nouveaux supports dans l'enseignement de la littérature. Au même titre que les autres arts : la littérature, la peinture, la photographie, le cinéma aussi doit être considéré comme un support pertinent grâce auquel l'apprenant peut apprendre tout en s'épanouissant. Le cinéma devient un moyen d'écriture : la mise en scène n'est plus un moyen d'illustrer ou de présenter une scène, mais une véritable écriture ; l'auteur écrit avec la caméra comme un écrivain écrit avec son stylo. D'après Alexandre Astruc, il n'est plus question de donner à voir la réalité littéraire, mais de faire entendre la voix d'un auteur. Pour lui, l'adaptation est comme un acte de création : La « caméra est un "stylo" grâce auquel le cinéaste, à l'égard du romancier, pourrait exprimer sa pensée, aussi abstraite soitelle » (Astruc, 1948). Tout en essayant de rester fidele à l'auteur, le cinéaste pour Alain Garcia, suit les grandes lignes du roman et devient un traducteur dont l'objectif est double : traduire fidèlement tout en créant librement :

Quand un cinéaste parvient à se hisser au niveau du romancier dans un contexte bien entendu de grande littérature, lorsque ses qualités d'adaptateur ou de transposeur (en collaboration avec son scénariste, il va de soi) lui permettent de se rapprocher de l'œuvre initiale, parfois même de rivaliser de brio avec elle sur le plan de la fidélité à la lettre et à l'esprit. Le cinéaste peut alors réussir à produire une « œuvre double », au même niveau que l'originale. Mais c'est aussi plus qu'une transformation, une véritable métamorphose cinématographique d'une œuvre qui jusque là n'était que romanesque. (...) Dans le cas de cette approche spécifique qui n'est autre qu'une traduction filmique du roman, la littérature a tout à gagner et rien à perdre. Traduire une œuvre littéraire - de langue à langue - est un travail qui, indéniablement, va dans le sens de la littérature et de la culture ; il en est de même dans le cas d'une analogie qui est une traduction de langue à langage, mais qui possède les mêmes qualités esthétiques, culturelles ou idéologiques que sa devancière, devenant par là-même aussi indispensable qu'elle ». (Garcia, 1990: 250).

Par contre, Chabrol affirme avec un certain orgueil que le film est supérieur à la littérature dans sa capacité de faire entendre certains sons qui, évoqués par l'auteur du roman, ne résonnent pas chez le lecteur. Si le principe est incontestable, l'exemple dont il se sert pour prouver son hypothèse ne l'est pas. Parlant des éléments musicaux du roman, Chabrol mentionne:

La sonate de Scarlatti qu'Emma joue puis s'arrête de jouer par lassitude ». «Et là », dit-il, «j'ai un avantage incontestable sur Flaubert, c'est que je peux la faire entendre, que mon spectateur peut rêver dessus, tandis que dans le texte, le lecteur n'a qu'un nom, la sonate de Scarlatti : il la connaît ou il ne la connaît pas. (Chabrol, 1991: 60).

Or, dans le texte de Flaubert, nulle mention de la sonate de Scarlatti. C'est donc une invention du cinéaste, et son lapsus prouve jusqu'à quel point cet ancien auteur de la Nouvelle Vague s'est confondu avec Flaubert lui-même. Par sa capacité à faire entendre plusieurs sons à la fois, le cinéma l'emporte également sur le roman dont les 
descriptions se déroulent forcément dans le temps. Alors que le romancier doit compter sur l'imagination du lecteur pour rendre simultané ce qui est évoqué de façon successive dans sa prose, le cinéaste peut dans la postproduction du film « mixer » les diverses pistes sonores pour faire entendre en même temps voix, musique, bruits.

Les adaptations cinématographiques doivent rester fidèles au texte comme la traduction par rapport au texte source préserver le même effet sur le lecteur (Lomaka, 2017 : 234) et donner à l'audio-spectateur du film une nouvelle appréciation du texte littéraire.

Les ouvrages théoriques et pratiques étant nombreux sur ce sujet, il ne s'agit pas dans cet article de proposer de nouvelles théories mais une réflexion qui permettra aux étudiants de mieux porter leurs jugements grâce aux supports audio-visuels.

\section{Différentes adaptations de Madame Bovary au cinéma}

Les adaptations d'œuvres littéraires sont nombreuses au cinéma. Dans le travail de l'adaptation, il s'agit de créer un récit cinématographique à partir du récit littéraire. Madame Bovary est un des romans les plus adaptés au cinéma. On en compte dix-huit adaptations en France et dans le monde entier: en Pologne, en Angleterre, en Allemagne, au Portugal, en Argentine, en Russie, aux États-Unis. Cette étude se réfère aux adaptations cinématographiques du roman de Flaubert par :

Jean Renoir, 1933,

Vincente Minnelli, 1949,

Claude Chabrol, 1991, et

Sophie Barthes, 2014.

La première adaptation cinématographique de Madame Bovary date de 1932. Un an plus tard 1933, la version de Jean Renoir est très mal accueillie en France. En effet, la version originale durant plus de trois heures, les distributeurs ont été obligés de couper le film et, ce faisant, l'ont en partie dénaturé. Valentine Tessier (1892-1981) incarne Emma Bovary dans l'adaptation de Jean Renoir (1933). Elle est en effet imposée par le producteur Gaston Gallimard qui est alors l'amant de Valentine Tessier.

En 1990, Claude Chabrol réalise une adaptation fidèle de Madame Bovary et vise qu'aucune scène ne soit tournée en studio. Il affirme qu':

Il fallait que j'arrive à le faire pour pouvoir continuer à me regarder dans la glace. C'est une nécessité profonde, parce que Madame Bovary correspond à mon rêve d'œuvre d'art... Il y a très peu de dialogues dans le livre, mais tous les dialogues du film sont des phrases de Flaubert. J'ai travaillé sous l'œil de Gustave, un portrait qui me regarde tantôt avec bienveillance tantôt avec sévérité. (Chabrol, 1991)

Isabelle Huppert (née en 1953) tient le rôle d'Emma Bovary dans la version, qui se veut fidèle au roman, de Claude Chabrol (1991). La complicité entre l'actrice et le metteur en scène donnera lieu à de nombreuses collaborations. Le film est notamment tourné au château de Breteuil, à Versailles et à Rouen. La voix off est celle de François Périer. Jean-François Balmer incarne Charles Bovary, Christophe Malavoy Rodolphe, Lucas Belvaux Léon et Jean Yanne le pharmacien Homais. Le scénario est de Claude Chabrol. L'actrice, Isabelle Huppert représente à ses yeux l'incarnation idéale d'Emma. Elle-même analyse ainsi son interprétation du personnage :

J'ai cherché à restituer au personnage sa vraie violence, sa perversité aussi et, surtout, ce que j'ai trouvé dans le roman de Flaubert, son sens aigu de la dérision, trois vertus sans lesquelles Emma ne serait qu'un légume, qu'une

XLinguae, Volume 12, Issue 3, June 2019, ISSN 1337-8384, eISSN 2453-711X 
molle provinciale alanguie. Je me sens plus proche, d'une certaine manière, de Flaubert que d'Emma, du créateur que de son archétype. (Boulat, 1991)

Trois des films du corpus se servent d'une voix off qui assiste la narration du film. En effet, l'adaptation de Renoir n'utilise pas de voix narratrice, car cela ne se faisait pas encore, cinq ans après la naissance du parlant. Chez Chabrol, alors qu'Emma résiste à la tentation d'acheter les tissus de Lheureux, elle s'efforce d'être une parfaite maîtresse de maison, et lavant les bottes de son mari, la voix off raconte : " Quand Charles rentrait, il trouvait près des cendres ses pantoufles à chauffer, ses gilets ne manquaient plus de doublure et ses chemises de boutons, elle ne rechignait plus comme autrefois à faire des tours de jardin ». La voix off devient alors un relais à la narration, elle marque la répétition du quotidien d'Emma dans le film de Chabrol et place en fond de champ, par la voix off. Par ailleurs, la voix off accélère le temps, comme chez Minnelli au moment de la convalescence d'Emma suite à la rupture de Rodolphe, Flaubert raconte L'été disparut. L'hiver succéda à l'automne, apportant l'oubli, pendant qu'à l'écran, Emma passe de son lit à son fauteuil à bascule, les saisons défilant à sa fenêtre. Chabrol marque des ellipses temporelles, comme par exemple la saute entre la rencontre d'Emma avec Rodolphe au cabinet de Charles et les Comices, ils arrivèrent en effet ces fameux Comices dit-elle.

En effet, la voix du narrateur en off du personnage de Flaubert essaie de faire comprendre au lecteur/spectateur la manière de penser d'un personnage. Le narrateur, la voix off commente et raconte dans les détails beaucoup de passages non montrées. Les réalisateurs trouvent que le texte de Flaubert est facile à réaliser :

Ce qui est fantastique chez Flaubert, c'est qu'il a déjà conçu le scénario ; quand on adapte l'une de ses œuvres au cinéma, il suffit de respecter ce qu'il a fait pour être sûr de tomber juste et de rester fidèle au roman. Il est précis dans les détails de mise en scène. (Alexandre, 2003: 54)

\section{L'incipit du texte et la réalisation en support audio-visuel}

L'incipit du texte offre, en général, des réflexions sur son utilisation future, en particulier dans les domaines liés aux langues, aux médias ou à l'éducation interculturelle (Perez, 2015 :28). Dans l'incipit de Madame Bovary, Flaubert nous présente une scène théâtrale où est dressé le portrait de l'antihéros, Charles Bovary, futur époux d'Emma. Le personnage de Charles est présent dès la première phrase en tant que «nouveau», et exposé à toute la classe. Charles a quinze ans quand commence le roman. Son portrait est présenté par de nombreuses descriptions sur son aspect physique et sur son attitude. Bien qu'il soit plus âgé que ses camarades de classe, il apparaît mal à l'aise tout au long du passage : « Nous avions l'habitude, en entrant en classe, de jeter nos casquettes par terre ». (Flaubert, 1857). La description du portrait et de la personnalité de Charles offre au lecteur des indices sur le monde dans lequel va évoluer la vie d'Emma Bovary.

En choisissant pour cadre l'intérieur de la classe, l'illustrateur met au premier plan Charles et sa casquette. Par contre les quatre adaptations cinématographiques, la version de Renoir, de Minnelli, de Chabrol et de Barthes ont ignoré les premières pages du roman. Ils n'ont pas montré l'enfance du personnage de Charles. Voici comment les quatre réalisateurs ont procédé pour le début de leur film : l'adaptation de Renoir commence par une présentation idyllique de la ferme avec des cochons et des vaches qui frôlent sous les arbres et des servantes qui étendent le linge au soleil. Ensuite la caméra nous montre l'intérieur sombre du salon où Emma entretient Charles en lui montrant un portrait de Stuart. Emma voit Charles comme son sauveur et rêve déjà d'une vie romantique. Même si Renoir affirme dès la première scène les rêves et des aspirations d'Emma en contradiction avec sa vie à la ferme : alors qu'elle 
souhaiterait vivre à l'époque de Marie Stuart, elle s'occupe de la traie de la truie entre autres. Elle se sent étriquée, à l'image de ses robes trop moulantes.

De toutes les adaptations du roman à l'écran, seule la version de Vincente Minnellimet en scène le procès de Flaubert, à l'ouverture et à la clôture du film. Cette version raconte l'histoire du livre par Flaubert défendant son roman devant un tribunal. Le réalisateur a placé l'auteur parmi les personnages et c'est grâce à cette scène que le spectateur reçoit le message du roman. Minnelli procède par un flashback sur les années passées d'Emma au couvent juste après l'apparition d'Emma dans la cuisine sale des Bertaux en belle robe blanche brodée. Cette apparition montre bien qu'elle est en désaccord avec le monde paysan dans lequel elle vit.

Claude Chabrol choisit de modifier quelques éléments du roman de Flaubert. Le film commence sur la scène de la rencontre entre Charles et Emma. En tant que spectateur, Charles dans le film est présenté comme un homme timide : on retrouve la fameuse réplique provenant de l'incipit du roman, «Charbovari» dans le film, lorsqu'Emma lui demande son nom. De plus, elle lui demande de répéter, comme l'avait fait le maître d'école dans le livre. Il va soigner M. Rouault, mais les plaisanteries vis-à-vis du patient disparaissent dans le film. Dans une autre scène avec Emma près de sa fenêtre, le personnage de Charles bégaie et n'articule pas ses mots, ce qui le rend inaudible. Cela reflète parfaitement le Charles décrit dans l'incipit de l'œuvre de Flaubert, c'esta-dire un jeune homme peu sûr de lui, timide et gêné. Sophie Barthes a choisi de mettre la scène de la mort dès le début.

... cette idée de tout faire en flashback et que ça commence et finisse par la mort est venue au montage. Du coup, quoiqu'elle fasse, y compris des choses aussi anodines qu'acheter un bout de tissu, ça la mène vers sa mort... J'ai choisi de ne pas la faire mourir dans son lit, comme dans le livre, mais de la ramener à la nature. (Pierrette, 2015).

\section{Le destin de Madame Bovary}

Les quatre films racontent tous, de manière chronologique, la même histoire, celle d'Emma Bovary, de son mariage avec Charles jusqu'à sa mort, en passant par ses relations extraconjugales et son suicide qui clôt chaque récit. Ces grandes scènes qui constituent le roman restent dans la mémoire du lecteur: la noce, le bal de la Vaubyessard, l'opéra de Rouen et le bal masqué, les comices et la mort d'Emma. Surtout le bal de la Vaubyessard, les Comices agricoles et l'opéra sont importantes aux yeux d'Emma, elles ponctuent sa vie parce qu'ils sont uniques. Dans un premier temps, l'éducation d'Emma dans l'institution religieuse va jouer beaucoup sur sa vie. Dans son essai sur Madame Bovary, Micheline Hermine explique la situation de la femme au XIXe siècle. L'éducation religieuse isole les filles du monde, les oriente vers la rêverie, enseigne le mépris du corps, le dégoût de la sexualité, les détourne de la vie. (Hermine, 1997:175).

L'éducation d'Emma au couvent est reprise dans les quatre adaptations : Chez Renoir cette séquence est prise en charge par un dialogue, par la voix-off chez Chabrol tandis que chez Minnelli les quelques séquences de couvent ne montrent pas une Emma lectrice :

Pendant six mois, à quinze ans, Emma se graissa donc les mains à cette poussière des vieux cabinets de lecture. "Dans la version de Sophie Barthes, Emma sort du couvent pour se marier et prie Dieu pour « que ce soit le bon » contrairement au roman de Flaubert : "Emma, rentrée chez elle, se plut d'abord au commandement des domestiques, prit ensuite la

XLinguae, Volume 12, Issue 3, June 2019, ISSN 1337-8384, eISSN 2453-711X 
campagne en dégoût et regretta son couvent. Quand Charles vint aux Bertaux pour la première fois, elle se considérait comme fort désillusionnée, n'ayant plus rien à apprendre, ne devant plus rien sentir. (Madame Bovary, 1ère partie, chapitre 6).

\section{Les noces}

Deux jours après la noce, les époux s'en allèrent, et aussitôt, dès le rendement, Emma se sent déçue par le mariage avec Charles, mari[...] sans grand emportement, elle lui reprochait de n'être qu'un personnage ordinaire, médiocre même : "La conversation de Charles était plate comme un trottoir de rue, et les idées de tout le monde y défilaient dans leur costume ordinaire, sans exciter d'émotion, de rire ou de rêverie ». (Ibid., 1ère partie, chapitre 4).

On relève dans toutes les versions cinématographiques des plans d'Emma à la fenêtre et des scènes de repas qui expriment à la fois la solitude et la claustration.

\section{La séquence du bal}

Le bal raconte un épisode très attendu d'Emma. Il s'agit d'une rupture avec la monotonie et l'ennui de sa vie, mais ce moment signifiera pour elle le début d'une grande déception. Ce moment est raconté à travers le point de vue d'Emma, qui observe la salle de bal depuis la porte, en scrutant la mise des autres femmes, puis c'est son émotion qui est décrite lorsqu'elle commence à danser. Le lecteur découvre au fur et à mesure ses moindres pensées et les moindres détails observés.

Tous les membres du bal apparaissent comme des êtres d'exception. L'impression que lui procure le bal est placée sous le signe de l'hyperbole « aux fulgurations de l'heure présente », «s'évanouissait toute entière, elle doutait presque de l'avoir vécu ». L'antithèse entre le présent et le passé rappelle qu'Emma n'appartient pas au monde qui évolue dans ce bal.

La séquence du bal de la Vaubyessard est centrale dans le roman comme dans toutes les adaptations. Elle constitue un point névralgique à partir duquel Emma va basculer et se perdre irrémédiablement dans ses rêves et ses chimères. Emma semble plus intéressée par ce que les autres disent que par la soirée. Mais elle ne prend pas part aux discussions, elle est une étrangère dans un monde qui n'est pas le sien mais semble flotter comme dans un rêve. Puis un événement insolite l'arrache brusquement à sa rêverie : un domestique casse des vitres avec une chaise pour rafraîchir l'air «lourd » de la salle de bal. Buvant du champagne, dansant avec un Vicomte, Emma vit ses rêves pendant plusieurs heures. Dès qu'Emma se met à danser, son visage devient radieux, et sa honte sociale, ressentie jusqu'alors est déplacée sur son mari, définitivement gauche et maladroit, incapable de s'intégrer aux danses. L'ivresse marquée d'Emma chez Chabrol est focalisée par un gros plan sur les robes qui tournoient avec le rythme de la valse : «Ils commencèrent lentement, puis allèrent plus vite. Ils tournaient : tout tournait autour d'eux ». (Madame Bovary, Ire partie, chapitre 8).

C'est chez Minnelli que cette séquence est la plus développée. Il la voit peut-être comme un miroir des grandes parades hollywoodiennes. Emma y est sous le regard des hommes, séducteurs et prédateurs, et celui admiratif de son mari qui pourtant s'ennuie et boit plus que de raison. Pour Minnelli, le bal sert à Emma de vivre son rêve dans un système hollywoodien qui revendique le spectaculaire, et faire sa rencontre avec Rodolphe de manière spectaculaire. De même, dans la version de Chabrol, c'est au bal que la rencontre a lieu avec Rodolphe, alors que dans le roman, elle se fait à l'occasion d'une visite du baron de la Huchette au médecin pour la saignée d'un de ses fermiers. 
Quant à l'adaptation de Sophie Barthes, la disparition du bal de la Vaubyessard est une volonté de ne pas s'attarder sur le bovarysme d'Emma, déjà exprimé rapidement dans les quelques séquences où Emma feuillette des keepsakes. Puisqu'elle ne peut pas vivre dans sa vie présente, elle cherche l'idéal espérant rencontrer quelqu'un qui complétait ses sentiments et ses désirs pour être satisfaite. Elle fuit donc de son ennui en prenant le chemin de l'adultère. Installé à Yonville le couple Bovary espère trouver une vie qui plaira à Emma. Ses rencontres avec M. Lheureux, habile commerçant, le Marquis d'Andervilliers, et Léon, jeune clerc de notaire, vont rompre la monotonie de son existence. Emma se lie d'amitié avec Léon, l'amoureux tendre, faible, médiocre qui a certes plus de qualités que Charles mais ne vaut pas mieux.

Rouen marque ensuite une nouvelle étape dans la découverte des plaisirs des sens pour Emma : pour se fréquenter en secret, le jeune couple investit une chambre de l'Hôtel de Boulogne qui devient le lieu de leurs ébats dans toute la troisième partie. Emma passe ainsi du rêve d'un amour idéal à une passion vécue et charnelle. Leur premier rendez-vous, pour une visite de la cathédrale, les voit devenir amants au cours d'une promenade en fiacre. Rideaux fermés, la voiture parcourt la ville et les environs en tous sens, pendant des heures. Cet épisode fameux est de ceux qui servirent à l'accusation de Flaubert dans le procès intenté à son roman pour outrage aux bonnes mœurs et à la religion. Pourtant, la suprême habileté du romancier « réaliste » est ici de ne rien décrire de ce qui se passe à l'intérieur du fiacre.

Les amours d'Emma et Léon dans la troisième partie du roman sont une autre occasion pour les illustrateurs de mettre en scène l'érotisme du personnage. En choisissant pour cadre l'intérieur de la chambre d'hôtel dont les volets restent clos, nous partageons l'intimité des deux jeunes gens : Emma se révèle alors dans toute sa beauté sensuelle. La description de Flaubert nous permet de contempler cette scène : «Ce furent trois jours pleins, exquis, splendides, une vraie lune de miel. Ils étaient à l'hôtel de Boulogne, sur le port. Et ils vivaient là, volets fermés, portes closes, avec des fleurs par terre et des sirops à la glace, qu'on leur apportait dès le matin ». (Madame Bovary, p.339)

Le premier amant d'Emma est Rodolphe. Rodolphe est un homme qui sait parler aux femmes, qui comprend Emma et qui, ayant peur de l'engagement, comprend surtout qu'il se doit de la fuir. Chez Minnelli, sa fuite est justifiée d'une manière un peu différente, puisqu'il apparaît comme un véritable amoureux obligé de fuit une maîtresse exigeante, dévoreuse, menaçante et perverse. Contrairement au roman, dans le film de Minnelli, Emma rencontre Rodolphe à la Vaubyessard, dans le rôle du vicomte, ce qui a pu donner l'idée de fondre dans un même personnage, le vicomte, le marquis et Rodolphe. Ce personnage est un beau parleur chez les quatre réalisateurs. Assommée de dettes, Emma supplie Rodolphe de lui prêter de l'argent. N'étant qu'un profiteur et un consommateur de femmes, il la renvoie promener de manière impassible en frôlant l'ignominie : "Je ne les ai pas, chère madame ». (Ibid., p 557), dit-il d'un air très calme. Tandis qu'Emma devient une proie fragile, elle s'agite dans tous les sens exerçant une chorégraphie angoissée du va-et-vient.

\section{La mort d'Emma}

Emma se sent très seul et souffre beaucoup de la solitude comme le Robinson de Defoe isolé sur l'île de Speranza (Veverkova, 2014 :28). Ce qui lui manque beaucoup, c'est la présence de son amant, très importantes à ses yeux. Elle préfère donner fin à ses jours. Et toutes les versions cinématographiques insistent sur l'agonie d'Emma, et son rapport à la religion notamment quand elle baise le crucifix.

La veillée funèbre d'Emma Bovary offre, un exemple de respect de l'illustrateur envers l'œuvre littéraire: "La cire des cierges tombait par grosses larmes sur les draps du lit. Charles les regardait brûler. » Madame Bovary, 3 partie, chapitre 9.

XLinguae, Volume 12, Issue 3, June 2019, ISSN 1337-8384, eISSN 2453-711X 
L'auteur met en scène l'abbé Bournisien et le pharmacien Homais somnolents autour du lit de la défunte :

Ils étaient en face l'un de l'autre, le ventre en avant, la figure bouffie, l'air renfrogné, après tant de désaccord se rencontrant enfin dans la même faiblesse humaine ; et ils ne bougeaient pas plus que le cadavre à côté d'eux qui avait l'air de dormir. / Charles, en entrant, ne les réveilla point. C'était la dernière fois. Il venait lui faire ses adieux. (Ibid., p.575).

Bien que Madame Bovary soit au centre de l'attention, les trois personnages masculins sont mis en premier plan. La tête penchée en avant de Monsieur Bovary et la position de l'abbé et du pharmacien endormis, montrent bien l'immobilité de l'homme à résoudre les problèmes, l'homme est spectateur de sa propre tragédie. Chez Renoir et Chabrol, l'agonie d'Emma est un supplice affreux (pour le spectateur aussi) qui s'achève sur le rire terrible et empreint de lucidité d'Emma ; au contraire tandis que chez Minnelli, l'héroïne reste magnifique, le réalisateur embellit la mort d'Emma. La chute d'Emma, de la prise de l'arsenic à sa mort, la plus longue est dans l'adaptation de Renoir, soit neuf minutes, chez Chabrol et Minnelli elle meurt en six minutes.

Renoir réalise l'agonie d'Emma très touchante : La bouche et les yeux légèrement entrouverts, la tête sagement posée sur l'oreiller avec ses cheveux bien coiffés, Emma a simplement l'air de dormir ou de se reposer. Chabrol suit le texte de Flaubert à la lettre, mettant en scène les détails les plus prosaïques et les plus réalistes de l'agonie d'Emma, comme les cheveux défaits, le visage en sueur, la langue tout entière qui sort de la bouche et les vomissements. Si Emma a un affreux goût d'encre dans la bouche et se met à vomir du sang, Chabrol lui fera donc vomir de l'encre bleue ! Ce goût d'encre qu'elle a dans la bouche, c'est celui des livres qu'elle a ingurgités. Emma vomit ses lectures, elle subit une purgation de ses clichés (elle est purgée, dit M. Canivet). Les lectures sont donc convoquées à sa mort, comme elles ont façonné sa vie.

De plus, le gros plan sur le visage d'Emma à l'agonie est teinté de bleu. Cette couleur revêt aussi une dimension métaphysique qui vaut comme métaphore du passage et de la montée vers les cieux: Des gouttes suintaient sur sa figure bleuâtre, qui semblait comme figée dans l'exaltation d'une vapeur métallique.

Chez Sophie Barthes, on ne lit aucune référence à la religion : Emma meurt seule dans une forêt, mais sa mort n'est pas une agonie affreuse, malgré ses souffrances. Emma meurt essoufflée, comme elle a vécu, à courir après des hommes-chimères. La mort d'Emma est complètement différente de celle du roman. Les différences sont nombreuses : Emma ne s'empoisonne pas avec l'arsenic entreposé chez Homais (1h $44: 00)$ mais avec une fiole trouvée dans les affaires de son mari, elle va mourir, seule, loin du domicile conjugal, dans un chemin de forêt. Ainsi on ne voit pas défiler tout Yonville au chevet d'Emma, on n'y voit pas plus Charles éploré, ni Bournisien en train d'administrer les derniers sacrements, ni les docteurs Canivet et Larivière, ni Homais donner son repas. C'est seulement à la tombée du soir que Charles, accompagné de tous les villageois, part à sa recherche (1h.46:46). Cette métamorphose insiste sur la profonde solitude d'Emma au moment de mourir, ce qui se rapproche de l'esprit de Flaubert. Néanmoins la mort d'Emma dans le film est plus propre (pas de vomissures, de râles, de cheveux en bataille, de teint livide, de cernes profondes) que dans le roman. La robe d'Emma Bovary a « la couleur des feuilles, ce qui donne un effet caméléon où elle revient dans la terre et meurt comme un animal », affirme la réalisatrice. (Pierrette, 2015). 


\section{Conclusion}

L'image et l'adaptation cinématographique mettent l'accent sur les diverses techniques de l'écriture scénique, accompagnées de quelques suggestions pour une exploitation pédagogique susceptible d'aider l'enseignant à réaliser les objectifs escomptés en matière de l'enseignement de l'œuvre littéraire par le biais de l'outil audio-visuel. Mais, on a bien vu que l'adaptation cinématographique modifie et supprime des passages entiers du roman. De ce point de vue, on comprend mieux la méfiance de Flaubert à l'égard de l'illustration.

Même si les quatre adaptations de Madame Bovary racontent en effet la vie d'Emma, chaque réalisateur donne pourtant du personnage sa propre vision, son interprétation. La vision de Flaubert sur le monde qui l'entoure et sur les femmes dans les années 1850 ne paraît évidemment plus d'actualité lors des adaptations de son roman. La première d'entre elles, dont l'écart est soixante-seize ans, montre en effet que le réalisateur, quel qu'il soit, porte un autre regard sur le monde et sur le roman de Flaubert.

En effet, l'écart entre l'écriture du roman au XIXème siècle et sa première adaptation en 1933, soit soixante-seize ans plus tard, et cent trente-six ans avec celle de 1993 montre bien que des changements ont logiquement été opérés. Si Flaubert a donné des caractéristiques propres à Emma, les réalisateurs, étant donné qu'ils ont transformé tout ce qui l'entourait, modifient également et logiquement Emma pour en faire la leur. Ils ont leur propre vision du personnage de Flaubert, et leur image de la femme. Toutefois, les quatre cinéastes parviennent à l'indépendance totale grâce à leur propre point de vue. Le regard qu'ils portent sur le roman de Flaubert évolue au fil des décennies. Leur interprétation se modifie naturellement avec le temps, de même que les personnages changent d'un film à l'autre, et c'est surtout le portrait qu'ils font d'Emma qui se trouve le plus modifié, passant de la romantique à la solitaire puis à l'actrice. Si Flaubert voyait cela, sans doute il attaquerait en justice chaque illustrateur et réalisateur pour outrage à son livre. Mais, que ce soit le roman de Flaubert ou les adaptations cinématographiques, lecteur et spectateur trouvent un plaisir à la constitution d'éléments visuels et sonores réalisée par le passage du roman au film.

\section{Bibliographic references}

ALEXANDRE, W. 2003. Claude Chabrol : la traversée des apparences, Paris: Le Felin,ISBN: 2-86645-459-6.

ASTRUC, A. 1948. Naissance d'une nouvelle avant-garde », L'Écran français, 144, repris en mars 2018, www.newwavefilm.com/about/camera-stylo-astruc.shtml.

BOULAT, A. 1991.La (Sé)Vérité sans fards », Première, ${ }^{\circ} 169$.

CHABROL, C. 1991.Autour d'Emma, Hatier, Paris, ISBN: 978-2218039829.

VEVERKOVA, D. 2014. Le personnage de Vendredi dans Robinson Crusoé de Daniel Defoe et dans Vendredi ou les limbes du Pacifique de Michel Tournier, XLinguae Journal, Volume 7 Issue 2, ISSN 1337-8384.

FLAUBERT, G. 2001.Madame Bovary, édition présentée et annotée par Thierry Laget, « Folio classique », Gallimard, Paris.

GARCIA, A. 1990. L'adaptation du roman au film. I.F. Diffusion-Dujaric, Paris, ISBN : 9782859470456.

HERMINE, M. 1997. Destins de femmes, désir d'absolu, essai sur Madame Bovary et Thérèse de Lisieux. BEAUCHESNE, Paris.

LOMAKA, A. O. 2017. Literal approach to translation: a classification and literature review, in XLinguae, Volume 10, Issue 4, ISSN 1337-8384, eISSN 2453-711X.

PEREZ, E. 2015. Translation and Subtitling of Documentary Films in Teaching Foreign Languages and Intercultural Communication, in XLinguae Journal, Volume 8 Issue 1, ISSN 1337-8384.

XLinguae, Volume 12, Issue 3, June 2019, ISSN 1337-8384, eISSN 2453-711X 
PIERRETTE, M. 2015.Madame Bovary, ou comment faire un film d'époque dans "un marché absurde", Propos recueillis à Deauville le 11 septembre 2015.

TRUFFAUT, F. 1954. in Les Cahiers du cinéma, n 31, janvier 1954.

Available online :

Édition intégrale du manuscrit de Madame Bovary,http://www.bovary.fr/

Les adaptations:

https://flaubert.univ-rouen.fr/derives/mb_cinema.php

https://www.telerama.fr/cinema/films/madame-bovary,502623.php

www.allocine.fr/film/fichefilm-11/photos/detail/?cmediafile $=18821755$

Words: 5570

Characters: 34 467(19,15 standard pages)

Assoc. prof. dr. ValbonaGashi- Berisha

Department of French Language and literature

Faculty of philology, University " HasanPrishtina" of Prishtina

10000 Prishtina,

Kosovo

Valbonagashi@uni-pr.edu

Dr. LenditaGjikolli

Department of French Language and literature

Faculty of philology, University " HasanPrishtina" of Prishtina

10000 Prishtina,

Kosovo

Lendita.gjikolli@uni-pr.edu 